\title{
RESEARCH ON OPTIMAL OBSERVATION SCALE FOR DAMAGED BUILDINGS AFTER EARTHQUAKE BASED ON OPTIMAL FEATURE SPACE
}

\author{
Jin Chen ${ }^{1}$, Wenkai Chen ${ }^{1}{ }^{*}$, Aixia $\mathrm{Dou}^{2}$, Wen $\mathrm{Li}^{1}$, Yanping Sun ${ }^{1}$ \\ ${ }^{1}$ Institute of Lanzhou Earthquake Research, China Earthquake Administration, Gansu Lanzhou 730000, China \\ ${ }^{2}$ Institute of Earthquake Science, China Earthquake Administration, Beijing 100036, China
}

Commission III, WG III/1

KEY WORDS: Optimal Feature Space, Damaged Buildings, ESP, Scale, Accuracy

\begin{abstract}
:
A new information extraction method of damaged buildings rooted in optimal feature space is put forward on the basis of the traditional object-oriented method. In this new method, ESP (estimate of scale parameter) tool is used to optimize the segmentation of image. Then the distance matrix and minimum separation distance of all kinds of surface features are calculated through sample selection to find the optimal feature space, which is finally applied to extract the image of damaged buildings after earthquake. The overall extraction accuracy reaches $83.1 \%$, the kappa coefficient 0.813 . The new information extraction method greatly improves the extraction accuracy and efficiency, compared with the traditional object-oriented method, and owns a good promotional value in the information extraction of damaged buildings. In addition, the new method can be used for the information extraction of differentresolution images of damaged buildings after earthquake, then to seek the optimal observation scale of damaged buildings through accuracy evaluation. It is supposed that the optimal observation scale of damaged buildings is between $1 \mathrm{~m}$ and $1.2 \mathrm{~m}$, which provides a reference for future information extraction of damaged buildings.
\end{abstract}

\section{INTRODUCTION}

As an advanced technology of earth observation from space, remote sensing technology is used in the field of information extraction of earthquake damage because of its timeliness, economy, and the characteristics of simultaneous observation of a vast area. Damaged buildings are often the main factors causing casualties, therefore, the information extraction of damaged buildings based on remote sensing image is one of the research hotspots in the earthquake disaster assessment field and both domestic and foreign scholars have carried out a series of related research on the information extraction of damaged buildings. At the end of 20th century, some foreign scholars began attempting to detect earthquake damage information via spectral information and texture changes (Masashi Matsuoka, 1998b; Mitomi H, Yamazaki F, 2001b; Turker M.,2004a; Rathje E.M, 2005b). In recent years, with the rapid development of space remote sensing technology, the spatial resolution ratio of space remote sensing has been developed from $5 \mathrm{~m}$ (SPOT) to Sub-meter (Quickbird), and foreign scholars began to adopt the object-oriented seismic information extraction method. In 2005, T.Thuy Vu et al. experimented on the QuickBird image after the earthquake in Bam, Iran and extracted information of damaged building (T. Thuy $\mathrm{Vu}, 2005 \mathrm{~b}$ ). In 2015, Janalipour $M$ et al. used vector map before earthquake and full color sharpening high score image after earthquake for damage building detection, integrated classification results based on image elements and objects, and finally ascertained the damage degree of buildings according to the network-based fuzzy inference decision model (Janalipour M, 2015a). In 2016, F Yamazaki and W Liu used multi-source data (optical data on the airborne and satellite platforms) which was obtained after the 7.0-magnitude earthquake in Kumamoto, Japan, to extract and analyze the earthquake damage information. They compared the preliminary results with field investigation, global navigation system observation, and the seismic record, which worked well (F Yamazaki, 2016b).

Scholars of our country have also carried out related research, and made a series of important achievements. In the 1990s, the interpretation of earthquake damage was mainly visual interpretation, meanwhile, the extraction technology of aerial remote sensing information of earthquake damage gradually developed, so the flag of visual interpretation started to be established and the extraction method of earthquake damage information also developed from qualitative to quantitative, together with more attention paid to the investigation accuracy of earthquake damage (Zhang Decheng, 1993a). At the beginning of 21 st century, our country used aerial and satellite remote sensing data to obtain earthquake disaster information, which entered the practical stage (Liu Jiahang, 2004a). After 2006, the object-oriented information extraction method of earthquake damage was paid more and more attention to (Chen Wenkai, 2007b;Zhao Fujun, 2009a; Wang Huimin, 2011a). In $2016 \mathrm{Li}$ Qiang et al. compared the information extraction accuracy of damaged buildings by the fusion of various features through the image experiment and analysis after Yushu earthquake, via object-oriented method (Li Qiang, 2016a). Then the era walked to 2017, when Liu Ying et al. proposed an object-oriented method for detecting damaged buildings by integrating various kinds of characteristics such as shape, edge and corner points under an image segmentation framework (Liu Ying, 2017a).

\footnotetext{
* Corresponding author: Wenkai Chen; associate researcher;

E-mail: cwk2000@yeah.net.
} 
The remoting sensing information extraction method of damaged buildings after earthquake can be divided into two types: multi-temporal earthquake damage assessment method and mono-temporal earthquake damage assessment, the former affected by many factors such as data acquisition, sensor revisiting cycle, shooting angle and time, etc., with a larger difficulty in practical application, while the latter with less restriction, so it is relatively more effective to identify and extract damaged buildings directly by using remote sensing images after earthquake (Dong L, 2013a). Most of the previous researches were aimed at a certain earthquake case, data source or region, and part of the characteristic parameters of object's spectrum, geometry and texture characteristics were used during the extraction of damaged buildings via object-oriented method. This paper proposes an object-oriented extraction method of damaged buildings: generating multiscale objects based on ESP scale tool, optimizing the feature space by calculating the distance matrix and the minimum separation distance between various kinds of samples, and then using characteristic parameters which have been optimized to extract damaged buildings in the research area, as well as comparing with the traditional object-oriented method to test the efficiency and accuracy index of this method. In addition, this method is used to detect the optimal observation scale of damaged buildings as it is described in this paper.

\section{EXPERIMENTAL AREA AND DATA SOURCE}

Research data in this paper are remote sensing images of Jiegu Town, Yushu taken after the 2010 Yushu earthquake. The image data includes Quickbird image and UAV image after the earthquake, and the spatial resolution is $0.61 \mathrm{~m}$ and $0.2 \mathrm{~m}$ respectively. The Quickbird image has four wave bands (R, G, $\mathrm{B}, \mathrm{NIR})$, and the UAV image has three (R, G, B).
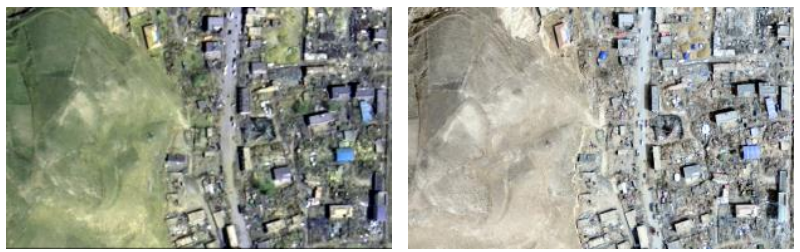

Figure 1 Research experimentation area (Quickbird image of Jiegu Town after the Yushu earthquake; UAV image of Jiegu Town after the Yushu earthquake)

\section{INFORMATION EXTRACTION OF DAMAGED BUILDINGS}

\subsection{Technical Route}

In this paper, the extraction process of damaged buildings consists of the following three steps: (1) Optimal- scale segmentation of objects: The extraction procedure established in this paper is based on the object-oriented extraction method. Since object generating is the first step, the remote sensing image is segmented by ESP (estimate of scale parameter) scale selection tool which can help us quickly get the optimal segmentation scale of the object; (2) Feature Space Optimization: we can calculate the distance matrix and the minimum separation distance between various kinds of samples through sample selection, and finally ascertain the optimal feature space of damaged buildings according to the minimum separation distance; (3) Information Extraction of Damaged Buildings: We extract the information of damaged buildings based on the optimal feature space which is obtained according to the above analysis, and then make accuracy evaluation through the comparison between extraction results and artificial interpretation. This paper specifically states the extraction procedure with Quickbird image of Jiegu Town after Yushu earthquake. The technical route is as shown in the Figure 2:

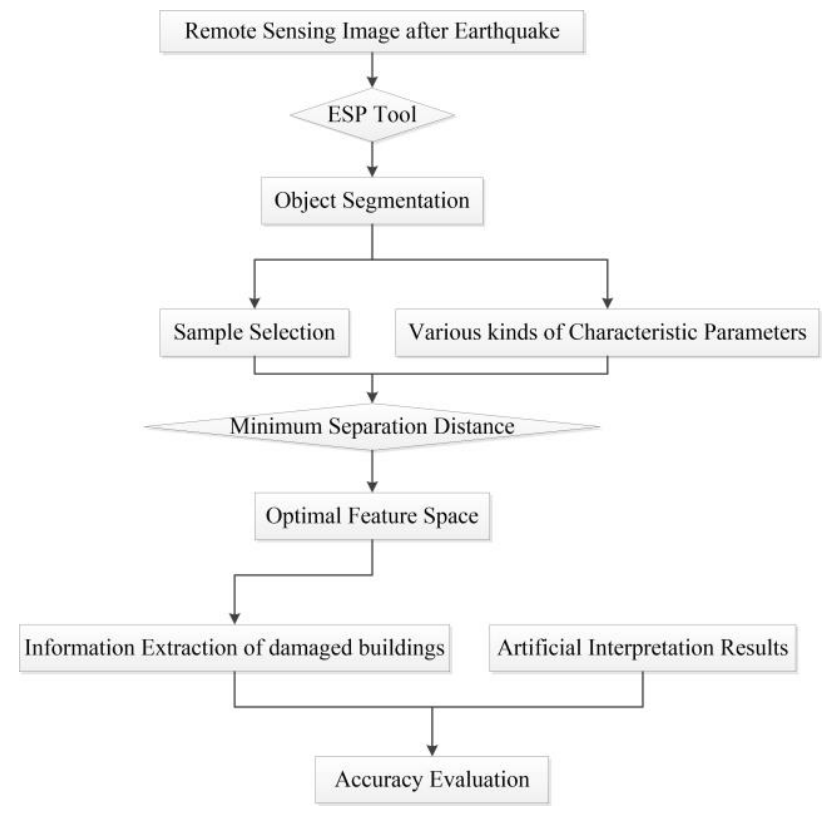

Figure 2 The extraction technical process of damaged buildings

\subsection{Optimal-scale Segmentation of Objects}

In this paper, the ESP tool, a kind of effective scale selection tool developed by Drăguţ (Drăguţ L, 2010a) et al. based on local variance, is used during the segmentation of objects, which can indicate whether the segmented objects have good consistency during object-oriented analysis for the special ability of local variance to well measure the regional homogeneity. This tool measures the rationality of scale through the mean value of local variance of segmented objects (LV) and rate of change of local variance of adjacent scale (ROC). The formula of ROC is as follows:

$$
R O C_{i+1}=\frac{L V_{i+1}-L V_{i}}{L V_{i}}
$$

In the formula, $\mathrm{LVi}+1$ represents the mean local variance of the ith plus first scale, LVi represents the mean local variance of the ith scale, and $\mathrm{ROCi}+1$ represents the rate of change of local variance during the change of segmentation scale from $i$ to $i+1$. The combination of the curves of ROC and LV can better show the meaningful change of segmentation result with the change of scale. The study shows that the optimal segmentation scale often appears in where the ROC-LV changes most rapidly (Chen Tieqiao, 2013a). When the ROC of the LV at a certain scale changes rapidly and then tends to flatten after the scale, it means that the segmentation result changes at that scale and the subsequent scale remains relatively stable. 'That scale' mentioned above is considered as the optimal segmentation scale. According to the above principle, use ESP tool to analyze the Quickbird image of Jiegu Town after Yushu earthquake (Figure 3), and we can see that the ROC before and after the segmentation scale of 55 meets the requirement, so the optimal 
segmentation scale for the experimental area is 55. From the segmentation result (Figure 4), we can see that intact buildings and completely extract their shadow, avoiding the confusion of intact buildings with damaged ones, so that the segmentation effect can be good.

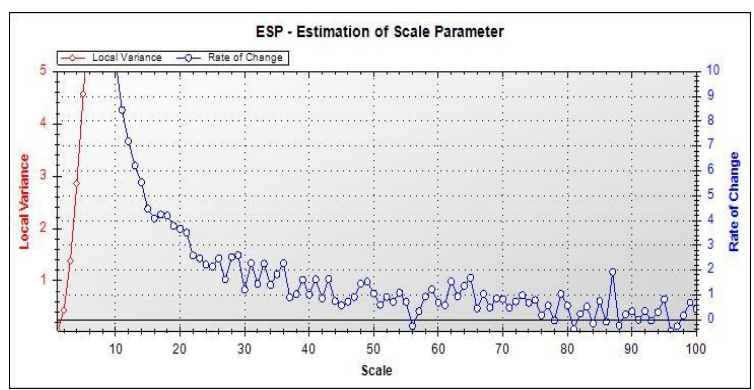

Figure 3 The curves of LV and ROC with the change of segmentation scale

\subsection{Feature Space Optimization-}

Through the analysis of the characteristics of remote sensing image in experimental area, it is found that the greyscale of the top of the basically intact buildings is equally distributed; after the building collapses, its top will become complicated and uneven due to the broken greyscale. The geometrical shape of the basically intact buildings is regular, mainly rectangle; after the building collapses, there will be either breakage/incomplete geometrical shape on its top, or large breakage/partial collapse/fall of corners due to the boundary ambiguity caused by partial collapse of the top of basically intact buildings. There are obvious texture characteristics on the top of basically intact buildings which is usually consistent with the direction of the boundary line; the collapse of the building destroyed the regularity of its structure and spatial layout in the original image, which are shown as characteristics such as contour disappearance and texture irregularity in the image, etc. The surface features around intact buildings are mainly road and trees on both sides of the road; after the building is damaged, there will be rubble or wall debris scattered along the road around the building. The paper mainly analyzes from several aspects such as spectral characteristics, texture characteristics and geometrical characteristics, etc. during the selection of initial characteristic parameters.

Spectral characteristics represent the spatial aggregation of spectral signature of surface features in the remote sensing images with different wave bands, sensors and time phases, mainly including greyscale, hue and color, etc. The parameters that reflect the spectral characteristics of damaged buildings after earthquake mainly include the mean value, the standard deviation, the ratio and luminance of each wave band. The texture characteristics of co-occurrence matrix is a texture description method on the basis of statistics, as well as a functional matrix of pixel distance and angle. It can reflect the comprehensive information of the image in direction, interval and rangeability by calculating the correlation between the two greyscales in a certain distance and direction of the image. The parameters that reflect the texture characteristics of damaged buildings after earthquake mainly include mean value, variance, contrast, entropy, homogeneity, dissimilarity, relativity, and angular second moment. The geometrical characteristics of buildings mainly refer to the shape of buildings and the characteristics based on geometrical relations. The parameters that reflect the characteristics of the geometrical shapes mainly include asymmetry, boundary index, compactness, density and Shape index, etc. In addition, NDVI and VDVI (Wang Xiaoqin, 2015a) are used for the better extraction of vegetation. NDVI is applied to Quickbird images, for the near-infrared band it contains, while VDVI is mainly applied to UAV images.

$$
\begin{gathered}
N D V I=(N I R-R) /(N I R+R) \\
V D V I=(2 \times G-R-B) /(2 \times G+R+B)
\end{gathered}
$$

According to the images of Jiegu Town after Yushu earthquake, we set up the extraction objects which can mainly be classified into five categories: Roads and Bare Land, Damaged Buildings, Intact Buildings, Vegetation and Shadow. Corresponding samples are selected for each type, according to which the separation distance between various kinds of surface features is calculated to get the minimum separation distance, and then to get the curve of change between the characteristic dimension and the minimum separation distance (Figure 4). From the curve in Figure 4, we can see that the classification effect will not get better as the number of characteristic value gets higher. Sometimes, there is redundancy because the number of characteristic value gets too high, which eventually leads to a decline in classification quality. When the characteristic dimension reaches 24 , the minimum separation distance reaches its maximum, and the classification effect is the best. After this, the minimum separation distance begins to decrease. However, when the characteristic dimension exceeds 15 , the slope of the curve obviously decreases, and the growth rate of separation distance obviously slows down. This paper only states the dimension of optimal feature space until 15 . The characteristic parameters in the finally- determined optimal feature space are NDVI, blue band mean value, compactness, greyscale, homogeneity of co-occurrence matrix, asymmetry, boundary index, green band ratio, red band ratio, near-infrared band standard deviation, density, near-infrared band ratio, Shape index, blue band ratio, and red band standard deviation. After calculation, the final classification distance matrix is as shown in Table 1, in which every category is well separated from each other, with a minimum separation distance of 2.496 .

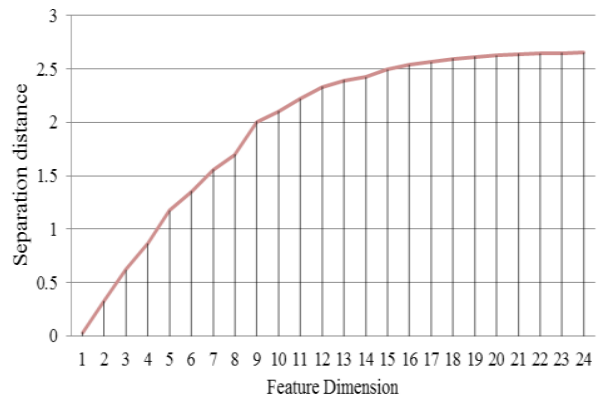

Figure 4 Curve map of separation distance and feature

\begin{tabular}{|c|c|c|c|c|c|}
\hline & $\begin{array}{c}\text { Damaged } \\
\text { Building } \\
\quad \mathrm{s}\end{array}$ & $\begin{array}{c}\text { Vegetatio } \\
n\end{array}$ & $\begin{array}{l}\text { Roads } \\
\text { and } \\
\text { Bare } \\
\text { Land }\end{array}$ & $\begin{array}{c}\text { Intact } \\
\text { Building } \\
\text { s }\end{array}$ & Shadow \\
\hline $\begin{array}{l}\text { Damaged } \\
\text { Buildings }\end{array}$ & 0 & 2.577 & 3.286 & 2.496 & 2.831 \\
\hline Vegetatio & 2.577 & 0 & 6.328 & 5.182 & 6.558 \\
\hline
\end{tabular}
dimension 


\begin{tabular}{cccccc}
\hline $\mathrm{n}$ & & & & & \\
$\begin{array}{c}\text { Roads } \\
\text { and Bare }\end{array}$ & 3.286 & 6.328 & 0 & 3.933 & 4.791 \\
$\begin{array}{c}\text { Land } \\
\text { Intact }\end{array}$ & 2.496 & 5.182 & 3.933 & 0 & 4.404 \\
$\begin{array}{c}\text { Buildings } \\
\text { Shadow }\end{array}$ & 2.831 & 6.558 & 4.791 & 4.404 & 0 \\
\hline
\end{tabular}

Table 1 Separation distance matrix

\subsection{Extraction of Damaged Buildings}

According to the 15 characteristic parameters in the optimal feature space obtained before, the information of the damaged buildings in the research area is extracted though a method called the nearest neighbor classification. The most similar sample object to each segmented image object is sought in the established optimal feature space, which is classified to the type of the sample object after being found. The distance between the image object $\mathrm{O}$ and the sample object $\mathrm{S}$ is calculated as follows:

$$
d=\sqrt{\sum_{f}\left(\frac{v_{f}^{(s)}-v_{f}^{(o)}}{\sigma_{f}}\right)^{2}}
$$

In the formula above, $\mathrm{f}$ represents the characteristic in the feature space, $V_{f}$ represents the corresponding characteristic value, $\sigma_{\mathrm{f}}$ represents the variance of the characteristic, and $\mathrm{d}$ represents the weighted Euclidean distance between the image object and the sample object. The final extraction result of damaged buildings is as shown in Figure 5. Make an accuracy evaluation by comparing the extraction result with the result of artificial interpretation (Table 2), you will get the overall accuracy of damaged building extraction which is $83.1 \%$, and Kappa coefficient 0.813. Landis and Koch have proved that when Kappa coefficient exceeds 0.80 , the consistency of the classification result will be very good (Landis J R, 1977a).

\begin{tabular}{ccccc}
\hline & $\begin{array}{c}\text { Production } \\
\text { Accuracy }\end{array}$ & $\begin{array}{c}\text { User } \\
\text { Accuracy }\end{array}$ & Kappa & $\begin{array}{c}\text { Overall } \\
\text { Accuracy }\end{array}$ \\
\hline $\begin{array}{c}\text { Extraction } \\
\text { Accuracy of } \\
\text { Damaged } \\
\text { Buildings }\end{array}$ & 0.872 & 0.774 & 0.813 & 0.831 \\
\hline
\end{tabular}

Table 2 Accuracy evaluation of extraction results based on optimal feature space

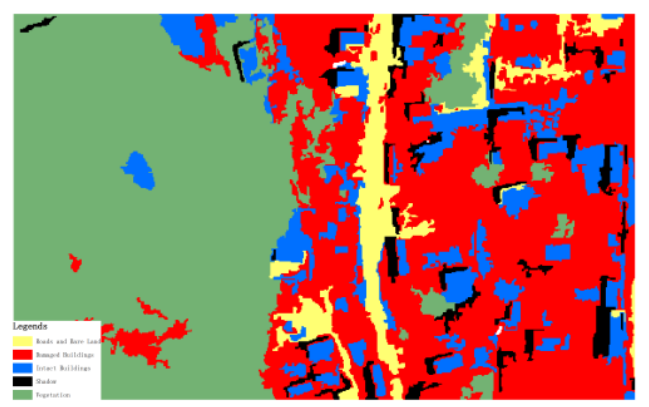

Figure 5 Extraction results based on optimal feature space

\subsection{Comparison Validation}

The traditional object-oriented method was often adopted in the previous research on the extraction of damaged buildings. Under most circumstances, by means of this method, we seek the optimal segmentation scale via experiment during image experiment, which takes much time. When classifying image objects, many people adopt the classification method based on decision tree, separating different types of surface features layer by layer and finally getting the damaged buildings according to the difference of individual characteristic values. Most of the characteristic values are also based on experience, which requires technicians to have rich image-analyzing experience and greatly influenced by human factors. The following is the extraction of damaged buildings in the same experimental area by traditional object-oriented extraction method. The extraction result is to be compared with that obtained by means of the method stated in this paper, to validate the efficiency and accuracy of the method stated in this paper.

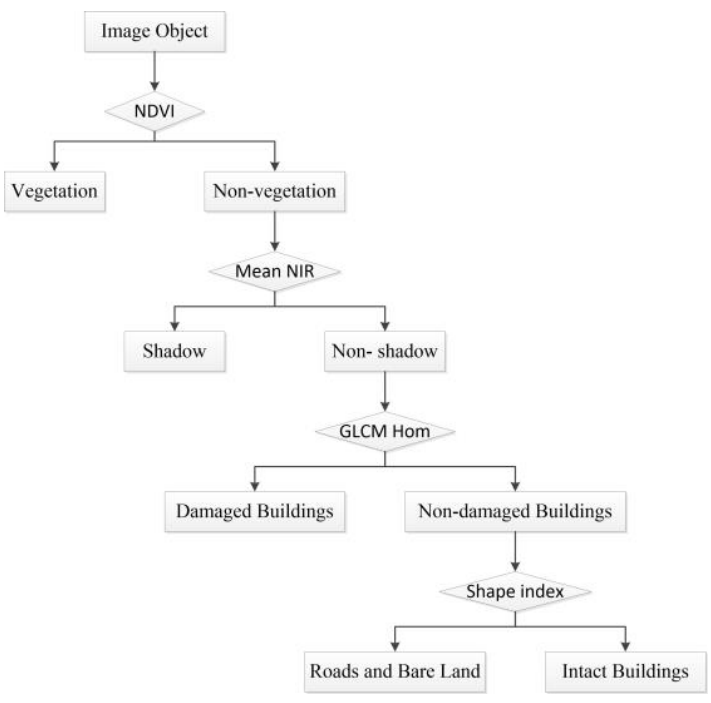

Figure 6 Classification decision tree

The segmentation scale is set as 40 according to previous study during object segmentation, and the extraction of damaged buildings is based on this scale (Zhao Y, 2016a). During the object classification, we use characteristic parameters like NDVI, Mean NIR, GLCM Homogeneity, and Shape index, etc. to establish a classification decision tree (Figure 6) and extract damaged buildings. Then we make accuracy evaluation through the comparison between extraction results and artificial interpretation (Table 3). The overall extraction accuracy reaches $75.9 \%$, the kappa coefficient 0.70 . It shows that the accuracy index of the extraction result via object-oriented extraction method based on the optimal feature space is better than that based on the traditional one. In addition, the extraction efficiency of the former method is also higher than that of the latter method. For the reason that the optimal scale and the optimal spatial feature in the procedure established in this paper are calculated automatically, and the characteristic parameters in the traditional object-oriented method needs to be artificially selected, the selection process of characteristic parameters will be of great difficulty and the extraction effect will not reach the expectation if without rich experience of image analysis as a support.

\begin{tabular}{llll}
\hline Production & User & Kappa & Overall \\
\hline
\end{tabular}




\begin{tabular}{lllll}
\hline & Accuracy & Accuracy & & Accuracy \\
\hline Extraction & & & & \\
$\begin{array}{l}\text { Accuracy of } \\
\text { Damaged }\end{array}$ & 0.798 & 0.690 & 0.700 & 0.759 \\
Buildings & & & & \\
\hline
\end{tabular}

Table 3 Accuracy evaluation of decision tree classification method

\section{SCALE INVESTIGATION OF DAMAGED BUILDINGS}

We extract damaged buildings after earthquake at different observation scales by the same extraction method. Here, we choose and study UAV image after Yushu earthquake to detect the change trend of the extraction effect of the damaged building as the scale changes, and speculate its optimal observation scale. Firstly, we resample the images to get a series of images of different spatial resolution, which is between $0.8 \mathrm{~m}$ and $1.6 \mathrm{~m}$, with an interval of $0.2 \mathrm{~m}$. Then we use ESP method to seek the optimal segmentation scale of different-resolution images and segment the images accordingly. When selecting samples among different-resolution images to establish the feature space, we'd better keep the number and spatial location of selected samples same in order to eliminate the interference caused by other factors. The number of the characteristics cannot determine the quality of the classification effect, so optimization of the characteristic by minimum separation distance is must before every classification, to establish the optimal feature space. Finally, we make accuracy evaluation on all the extraction results and then we can obtain the change trend of extraction effect of damaged buildings along with the change of observation scales. The evaluation Index is KIA (Kappa index of agreement), Hellden Accuracy, and Short Accuracy, among which Hellden Accuracy and Short Accuracy are the calculation result according to Production Accuracy and User Accuracy.

$$
H A=\frac{P A \cdot U A}{(P A+U A) / 2} ; \quad S A=\frac{P A \cdot U A}{P A+U A-P A \cdot U A}
$$

In the formula, HA represents Hellden Accuracy, SA represents Short Accuracy, PA represents Production Accuracy, and UA represents User Accuracy.

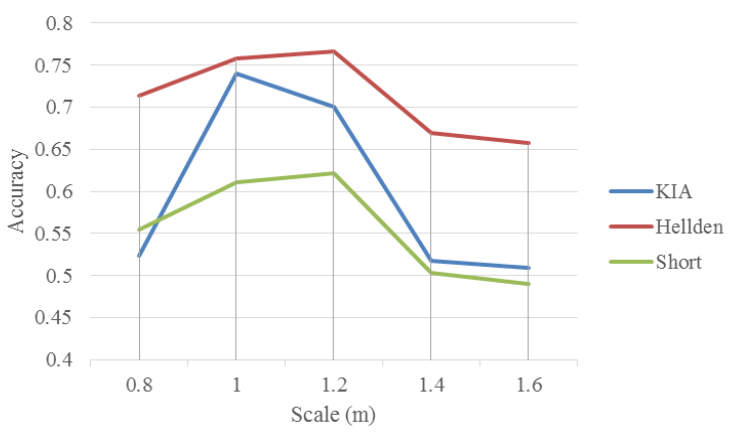

Figure 7 Trend graph of extraction accuracy with the change of scale

As is shown in Figure 7, when the observation scale changes, the extraction effect of damaged buildings changes accordingly. Through the observation of its changing trend, you can find the KIA curve and the SA curve peak at $1.2 \mathrm{~m}$, while the HA curve peak at $1 \mathrm{~m}$. Within the scale range between $1 \mathrm{~m}$ and $1.2 \mathrm{~m}$, there are both positive change factor and negative change factor existing, so we can speculate according to this that the optimal observation scale of damaged buildings is between $1 \mathrm{~m}-1.2 \mathrm{~m}$.

\section{CONCLUSION}

A new information extraction method of damaged buildings rooted in optimal feature space on the basis of the traditional object-oriented method is put forward in this paper, where several groups of high spatial resolution image after earthquake are validated and analyzed, as a result, the following points are obtained:

(1) The extraction method established in this paper adopts ESP tool to optimize the segmentation of image. Then the distance matrix and minimum separation distance of all kinds of surface features are calculated through sample selection to find the optimal feature space, which is finally applied to extract the image of damaged buildings after earthquake. The overall extraction accuracy reaches $83.1 \%$, the kappa coefficient 0.813 . The new information extraction method greatly improves the extraction accuracy and efficiency, compared with the traditional object-oriented method.

(2) The new method is used for the information extraction of different-resolution images of damaged buildings after earthquake, then to seek the optimal observation scale of damaged buildings. The research result shows that the extraction effect is now always better when space resolution gets higher, and optimal observation scale of damaged buildings is between $1 \mathrm{~m}$ and $1.2 \mathrm{~m}$.

(3) The extraction method established in this paper adopts computer automatic calculation during the selection of optimal scale and optimal feature space and there is almost no need for intervention of artificial experience, which reduces artificial subjective factors. This method greatly improves the extraction accuracy and efficiency, compared with the traditional objectoriented method, therefore, it owns a good promotional value in the information extraction of damaged buildings.

\section{REFERENCES}

Chen Tieqiao, Chen Jie, Mei Xiaoming, et al. 2013a. Field Theory Based Multi-level Clustering for High-Spatial Resolution Remote Sensing Imagery Segmentation. Geography and Geo-information Science, 29(6), pp:10-13.

Chen Wenkai. 2007b. Research on Remote Sensing Application Technology for Earthquake Damage Assessment. Institute of Lanzhou Earthquake Research, China Earthquake Administration.

Dong L, Shan L. 2013a. A comprehensive review of earthquake-induced building damage detection with remote sensing techniques. ISPRS J Photogramm, 84, pp:85-99.

Drăguţ L, Tiede D, Levick S R. 2010a. ESP: A tool to estimate scale parameter for multiresolution image segmentation of remotely sensed data. International Journal of Geographical Information Science, 24(6), pp:859-871.

F Yamazaki, W Liu. 2016b. Remote Sensing Technologies for Post-Earthquake Damage Assessment: A Case Study on The 
2016 Kumamoto Earthquake. Asia Conference on Earthquake Engineering.

Janalipour M, Mohammadzadeh A. 2015a. Building Damage Detection Using Object-Based Image Analysis and ANFIS From High-Resolution Image (Case Study: BAM Earthquake, Iran). IEEE Journal of Selected Topics in Applied Earth Observations \& Remote Sensing, 99, pp:1-9.

Landis J R, Koch G G. 1977a. An application of hierarchical kappa-type statistics in the assessment of majority agreement among multiple observers. Biometrics, 33(2), pp:363.

Li Qiang, Zhang Jingfa. 2016a. Research on Earthquake Damaged Building Extraction by Different Features Fusion. Journal of Seismological Research, 39(3), pp:486-493.

Liu Ying, Tao Chao, Yan Pei, et al. 2017a. Graph Cut Energy Driven Earthquake-damaged Building Detection from Highresolution Remote Sensing Images. Acta Geodaetica et Cartographica Sinica, 46(7), pp:910-917.

Liu Jiahang, Yang Jianfeng, Qin Xuwen. 2004a. Imagery Region Analyzing Method of Urban Building's Earthquake Damage: A Comparative Study with Point Characteristics-based Criterion Approaches. Journal of Natural Disasters, 13(2), pp:149-156.

Masashi Matsuoka, Fumio Yamazaki. 1998b. Identification of Damaged areas Due to the 1995 Hyogoken-Nanbu Earthquake Using Satellite Optical Images. Proceedings of the 19th Asian Conference on Remote Sensing, Q9, pp:1-6.

Mitomi H, Yamazaki F, Matsuoka M. 2001b. Development of automated extraction method for building damage area based on maximum likelihood classifier. Proceedings of 8th International Conference on Structual Safety and Reliability. California: New Beech. pp: 2430-3442.

Rathje E.M, Crawford M., et al., 2005b. Earthquake damage identification using multi-temporal high-resolution optical satellite imagery, IEEE International, 7, pp: 5045-5048.

T. Thuy Vu, M Matsuoka, and F Yamazaki. 2005b. Preliminary results in Development of an Object-based Image Analysis Method for Earthquake Damage Assessment. Proceeding of 3rd International workshop on Remote Sensing for Post-Disaster Response.

Turker M., San B. T., 2004a. Detection of collapsed buildings caused by the 1999 Izmit, Turkey earthquake through digital analysis of post-event aerial photographs. INT. J. Remote Sensing, 25(21), pp: 4701-4714.

Wang Huimin, Li Yan. 2011a. Object-oriented Damage Building Extraction. Remote Sensing Information, 26(5), pp:81-85.

Wang Xiaoqin, Wang Miaomiao, Wang Shaoqiang, et al. 2015a. Extraction of vegetation information from visible unmanned aerial vehicle images. Transactions of Chinese Society of Agricultural, 31(5), pp:152-159.
Zhang Decheng. 1993a. Preliminary Study on Visual Interpretation Marks of Building Damages Caused by Earthquakes on Aerophotograph. Earthquake, 1, pp:26-30.

Zhao Fujun, Zhang Lei. 2009a. Object-oriented Method for Extracting seismic Disaster Information Using Remote Sensing Images - A Case Study of Wenchuan Earthquake. Earthquake, 29, pp:130-138.

Zhao Y, Zhang J F, Yao L H. 2016a. Seismic damage information extraction and evaluation of buildings with high resolution remote sensing based on object-oriented method. Acta Seismologica Sinica , 38(6), pp:942-951. 